\title{
Definition of Healthy Ranges for Alanine Aminotransferase Levels: A 2021 Update
}

\author{
Luca Valenti (D) , ${ }^{1,2}$ Serena Pelusi, ${ }^{1,2}$ Cristiana Bianco (D),${ }^{2,3}$ Ferruccio Ceriotti, ${ }^{4}$ Alessandra Berzuini, ${ }^{2}$ Laura Iogna Prat, ${ }^{5}$ \\ Roberta Trotti, ${ }^{2}$ Francesco Malvestiti, ${ }^{1,2}$ Roberta D'Ambrosio, ${ }^{6}$ Pietro Lampertico iD , ${ }^{6,7}$ Agostino Colli, ${ }^{2}$ Massimo Colombo, ${ }^{8}$ \\ Emmanuel A. Tsochatzis (D) , ${ }^{5}$ Mirella Fraquelli, ${ }^{9}$ and Daniele Prati (iD 2
}

\begin{abstract}
The changing epidemiology of liver disease, and modifications in the recommended analytical methodology call for a re-evaluation of the upper reference limits (URLs) of alanine aminotransferase (ALT) levels. Using the same approach consolidated 20 years ago to define the healthy population, we defined the URL for the newly recommended International Federation of Clinical Chemistry (IFCC) standardized test. In a cross-sectional study, we examined 21,296 apparently healthy blood donors (age 18-65 years) and calculated the sex-specific URL by the 95th percentile in individuals without risk factors for liver disease. These were tested for the ability to predict liver damage in a subset of 745 participants with dysmetabolism, in an independent cohort of 977 unselected donors, and in 899 patients with chronic liver disease. ALT levels were measured by the IFCC test. Male sex, body mass index, glucose, lipids, ferritin, hypertension, and younger age were independent ALT predictors $(P<0.001)$. Updated URLs were identified at $42 / 30 \mathrm{U} / \mathrm{L}$ in males/females, approximately $30 \%$ lower than those currently recommended by the IFCC. Due to improved sensitivity, they conferred the ability to detect steatosis and significant fibrosis in individuals with dysmetabolism (odds ratio $[\mathrm{OR}]=2.31$, range $1.40-3.80, P=0.001 ;$ and $\mathrm{OR}=3.35$, range $1.19-9.42, P=0.021$; respectively), although with a limited accuracy, and significant fibrosis in unselected donors $(\mathrm{OR}=2.32,1.02-5.31, P=0.045)$. Updated URLs had a moderate to high accuracy to discriminate liver conditions (area under the receiver operating characteristic curve = 0.81, range 0.78-0.91). Conclusion: Updated URLs by the IFCC method were lower than those calculated in initial studies, but higher than those in use with the recommended old, nonstandardized method, and were able to better predict liver disease. The limited awareness that different techniques are still in use should be regarded as a possible source of medical errors. (Hepatology Communications 2021;5:1824-1832).
\end{abstract}

\section{SEE EDITORIAL ON PAGE 1807}

$\mathrm{S}$ erum alanine aminotransferase (ALT) is one of the most commonly requested biochemical tests in clinical practice and is an integral part of the diagnosis and management of patients with liver disease. Moreover, elevated ALT levels predict mortality independent of liver disease in the general population.
The most likely interpretation of this association is that nonalcoholic fatty liver disease (NAFLD), which is most frequently related to metabolic dysfunction (MAFLD), is now the leading cause of liver damage. ${ }^{(1-4)}$

In 2002, our group showed that the upper reference limits (URLs) for ALT used at that time were based on reference populations, which included a substantial proportion of individuals with subclinical liver disease, thereby limiting the sensitivity of ALT URLs to detect

Abbreviations: ALT, alanine aminotransferase; AUROC, area under the receiver operating characteristic curve; BMI, body mass index; CI, confidence interval; HBs Ag, hepatitis B surface antigen; HCV, hepatitis C virus; IFCC, International Federation of Clinical Chemistry and Laboratory Medicine; LR, likelihood ratio; LSM, liver stiffness measurement; $M / F$, males/females; NAFLD, nonalcoholic fatty liver disease; OR, odds ratio; URL, upper reference limit.

Received May 26, 2021; accepted July 2, 2021.

Additional Supporting Information may be found at onlinelibrary.wiley.com/doi/10.1002/hep4.1794/suppinfo.

Supported by Horizon 2020 Framework Programme (101016726 REVEAL and 777377 LITMUS), Associazione Italiana per la Ricerca sul Cancro (16888), Italian Ministry of Health (CV PREVITAL "Strategie di prevenzione primaria n" and "Ricerca Finalizzata RF-2016-02364358"), Associazione Italiana per la Prevenzione dell'Epatite Virale (COPEV), and IRCCS Ca' Granda Ospedale Maggiore Policlinico ("Ricerca Corrente" and 'Liver Bible' PR-0361").

(C) 2021 The Authors. Hepatology Communications published by Wiley Periodicals LLC on behalf of American Association for the Study of Liver Diseases. This is an open access article under the terms of the Creative Commons Attribution-NonCommercial-NoDerivs License, which permits use and distribution in any medium, provided the original work is properly cited, the use is non-commercial and no modifications or adaptations are made. 
liver injury. ${ }^{(5)}$ Thus, we proposed criteria for the definition of the reference population at low risk of liver disease by excluding carriers of viral hepatitis and individuals with metabolic syndrome and set the healthy thresholds for ALT at 30/19 U/L in males/females (M/F), respectively. The validity of this approach has been confirmed by studies focusing on the prediction of overall mortality $^{(6)}$ and liver-related events in the general population ${ }^{(7)}$, as well in individuals with biopsy-proven normal liver. ${ }^{(8)}$ Furthermore, the updated ALT thresholds have been widely adopted in clinical studies and guidelines.

To reduce variability of laboratory results, the International Federation of Clinical Chemistry and Laboratory Medicine (IFCC) promoted a standardization of the analytical methods in clinical enzymology, including that for ALT determination. ${ }^{(9,10)}$ The standardized method presents some technical differences when compared with those previously in use, the main one being a higher reaction temperature $\left(37^{\circ} \mathrm{C}\right.$ instead of $30^{\circ} \mathrm{C}$ ), which increases enzyme activity and results in higher ALT values. ${ }^{(9)}$ In addition, the upper thresholds recommended by this standardized method (59/41 U/L in $\mathrm{M} / \mathrm{F}$, respectively) were determined in a relatively small-size reference cohort, which consisted of individuals with risk factors for NAFLD. ${ }^{(11)}$

Thus, the modifications of the reference analytical methodology, and the changing epidemiology of the major causes of liver injury, with the rising role of $\operatorname{NAFLD~}^{(1-3,12,13)}$ call for a re-evaluation of the healthy ranges for ALT. The aim of this study was to examine the determinants and distribution of ALT by the IFCC method, to update the healthy ranges in a large cohort of apparently healthy individuals (ALT-URL-IFCC), and to validate their accuracy for detecting liver damage, in particular related to NAFLD. ${ }^{(14)}$

\section{Patients and Methods}

\section{STUDY COHORTS}

We considered 21,296 apparently healthy individuals, who presented for blood donation from May 2017 to April 2018 at the Transfusion Medicine and Hematology unit of the Fondazione Ca' Granda Hospital (Ca' Granda-2018 cohort). The enrollment criteria are reported in the Supporting Methods, and clinical features are presented in Table 1, upper panel. The study flow chart is presented in Supporting Fig. S1.

The updated ALT-URL-IFCC levels were estimated by the 95 th percentile observed in individuals $(n=9,195)$ without viral hepatitis (hepatitis B surface

View this article online at wileyonlinelibrary.com.

DOI 10.1002/hep4.1794

Potential conflict of interest: Dr. Prati advises and received grants from Macopharma. He received grants from Terumo, Ortho Diagnostics, Grifols, Immucor, Diamed, and Diatech. Dr. Lampertico advises and is on the speakers' bureau for Bristol Myers Squibb, Roche, Gilead, GlaxoSmithKline, MSD, AbbVie, Arrowhead, Alnylam, Eigar, Myr Pharma, and Janssen.

\section{ARTICLE INFORMATION:}

From the ${ }^{1}$ Department of Pathophysiology and Transplantation, Università degli Studi di Milano, Milan, Italy; ${ }^{2}$ Department of Transfusion Medicine and Haematology, Fondazione IRCCS Ca' Granda Ospedale Maggiore Policlinico, Milan, Italy; ${ }^{3}$ Department of Medicine, Udine Hospital, Udine, Italy; ${ }^{4}$ Clinical Laboratory, Fondazione IRCCS Ca' Granda Ospedale Maggiore Policlinico Milan, Milan, Italy; ${ }^{5}$ UCL Institute for Liver and Digestive Health, Royal Free Hospital and UCL, London, United Kingdom; ${ }^{6}$ Division of Gastroenterology and Hepatology, Fondazione IRCCS Ca' Granda Ospedale Maggiore Policlinico Milan, Milan, Italy; ${ }^{7} \mathrm{CRC}$ "A. M. and A. Migliavacca" Center for Liver Disease, Department of Pathophysiology and Transplantation, University of Milan, Milan, Italy; ${ }^{8}$ Liver Center, IRCCS San Raffaele Hospital, Milan, Italy; ${ }^{9}$ Department of Gastroenterology and Endoscopy, Fondazione IRCCS Ca' Granda Ospedale Maggiore Policlinico Milan, Milan, Italy.

\section{ADDRESS CORRESPONDENCE AND REPRINT REQUESTS TO:}

Daniele Prati, M.D.

Transfusion Medicine and Haematology

Fondazione IRCCS Ca' Granda Ospedale Maggiore Policlinico

Pad Marangoni, via F Sforza 35
20122, Milano, Italy

E-mail: daniele.prati@policlinico.mi.it

Tel.:+39-02-5503-4110 


\section{TABLE 1. CLINICAL FEATURES OF THE STUDY COHORTS (UPPER PANEL: 21,296 APPARENTLY HEALTHY INDIVIDUALS FROM THE CA' GRANDA-2018 COHORT, WHO WERE INCLUDED INTHE STUDY; LOWER PANEL: 745 INDIVIDUALS FROM THE LIVER-BIBLE-2020 COHORT)}

Ca' Granda-2018 Cohort, Whole

\begin{tabular}{lccc} 
Population & Males $(\mathrm{n}=13,741,64.5 \%)$ & Females $(\mathrm{n}=7,555,35.5 \%)$ & $P$ Value \\
\hline Age, years & $41.3(12.9)$ & $37.8(13.6)$ & $<0.0001$ \\
BMI, $\mathrm{kg} / \mathrm{m}^{2}$ & $24.9(3.2)$ & $23.0(3.6)$ & $<0.0001$ \\
Obesity, yes & $957(7.0)$ & $408(5.4)$ & $<0.0001$ \\
Glucose, $\mathrm{mg} / \mathrm{dL}$ & $86(10)$ & $83(12)$ & $<0.0001$ \\
Impaired fasting glucose, yes & $323(2.4)$ & $186(2.5)$ & 0.60 \\
Total cholesterol, $\mathrm{mg} / \mathrm{dL}$ & $184(35)$ & $182(33)$ & $<0.0001$ \\
Triglycerides, $\mathrm{mg} / \mathrm{dL}$ & $97(57)$ & $77(38)$ & $<0.0001$ \\
Arterial hypertension & $4,229(30.9)$ & $1,007(13.4)$ & $<0.0001$ \\
ALT, U/L & $26.2(13.3)$ & $247(3.3)$ & $<0.0001$ \\
HBsAg, positive & $251(1.8)$ & $246(3.3)$ & $<0.0001$ \\
Anti-HCV Ab, positive & $254(1.9)$ & $1,496(19.8)$ & $<0.0001$ \\
First-time donors & $1,902(13.8)$ & $<0.0001$
\end{tabular}

Ca' Granda-2018 Cohort, Population at Low Risk for Liver Disease Males $(n=4,855,64.5 \%)$ Females ( $n=4,340,35.5 \%)$

PValue

Age, years

$\mathrm{BMl}, \mathrm{kg} / \mathrm{m}^{2}$

Obesity, yes

Glucose, mg/dL

Impaired fasting glucose, yes

Total cholesterol, mg/dL

Triglycerides, mg/dL

Arterial hypertension

ALT, U/L

HBsAg, positive

Anti-HCV Ab, positive

First-time donors

Liver Bible-2020 Cohort

Age, years

BMI, $\mathrm{kg} / \mathrm{m}^{2}$

Obesity, yes

Glucose, mg/dL

Impaired fasting glucose, yes

Total cholesterol, mg/dL

Triglycerides, mg/dL

Arterial hypertension

ALT, U/L
37.1 (12.9)

$22.7(2.1)$$$
\begin{gathered}
0 \\
83(7)
\end{gathered}
$$

$83(7)$

0

$172(30)$

$73(26)$

$957(19.9)$

23.7 (12.8)

$$
0
$$

0

$786(16.4)$

$$
\begin{gathered}
35.8(13.0) \\
21.4(2.0) \\
0 \\
81(7) \\
0 \\
175(28) \\
67(24) \\
366(8.5) \\
18.0(8.4) \\
0 \\
0
\end{gathered}
$$$$
883(20.3)
$$

Females $(n=113,15.2 \%)$

Males $(n=632,84.8 \%)$

$$
\begin{gathered}
53.7(6.2) \\
29.0(3.5) \\
33(29.2) \\
94(13) \\
14(12.5) \\
211(32) \\
155(67) \\
82(72.6)
\end{gathered}
$$$$
31.5(13.9)
$$
0.88
0.21
0.12
0.15
0.010
0.001
0.018
0.58
$<0.0001$

Note: Data are shown as mean (SD) or frequency (\%). $P$ values were determined at univariate logistic regression models.

antigen [HBsAg], hepatitis B virus DNA, and hepatitis $\mathrm{C}$ virus [HCV]-RNA-negative), and with normal body mass index (BMI; $\leq 25 \mathrm{~kg} / \mathrm{m}^{2}$ ), cholesterol $(<240 \mathrm{mg} / \mathrm{dL})$, triglycerides $(<150 \mathrm{mg} / \mathrm{dL})$, and glucose $(<100 \mathrm{mg} / \mathrm{dL})$ levels, and without regular alcohol intake nor regular or recent recreational drug use. In a sensitivity analysis, we replicated exactly the criteria for ALT-URL definition in 2002, which differed from the main analysis due to the definition of dyslipidemia based on glucose $>105 / 98 \mathrm{mg} / \mathrm{dL}$ in $\mathrm{M} / \mathrm{F}$, 
triglycerides $>200 \mathrm{mg} / \mathrm{dL}$, and/or total cholesterol $>220 \mathrm{mg} / \mathrm{dL}$, and absence of medications. ${ }^{(5)}$

The diagnostic accuracy of the updated ALT-URLIFCC was validated in a subset of 745 individuals (a subset of the Ca' Granda-2018 cohort), consecutively enrolled from June 2019 to December 2020, who underwent noninvasive assessment of liver damage/ fibrosis due to the presence of dysmetabolism (at least three criteria among overweight [BMI $>25 \mathrm{~kg} /$ $\left.\mathrm{m}^{2}\right]$, arterial hypertension, hyperglycemia $[>100 \mathrm{mg} /$ $\mathrm{dL}]$, low high-density lipoprotein cholesterol $[<45 / 55$ in $\mathrm{M} / \mathrm{F}]$, and increased triglycerides $[>150 \mathrm{mg} / \mathrm{dL}]$ ), and age at risk (40-65 years). ${ }^{(15)}$ The clinical features of these individuals (Liver-Bible-2020 cohort) are reported in Table 1, lower panel. The presence of steatosis and of significant liver fibrosis were estimated by FibroScan with evaluation of continuous attenuation parameter $(\mathrm{CAP}>255 \mathrm{~dB} / \mathrm{m})^{(16)}$ and liver stiffness measurement $(\mathrm{LSM}>7.9 \mathrm{kPa}){ }^{(17)}$ performed within 4 weeks of ALT measurement. The updated ALT-URL-IFCC was further tested for the ability to predict increased LSM and significant liver fibrosis in a previously described independent cohort of 977 healthy blood donors from Northern Italy (Lecco cohort). ${ }^{(18,19)}$ Of these, 404 (41.7\%) were females, and mean age was $47.2 \pm 10$ years and BMI was $24.3 \pm 3.2 \mathrm{~kg} / \mathrm{m}^{2}$.

Additional validation cohorts in patients with liver diseases are reported in the Supporting Methods and Supporting Tables S1 and S2. ${ }^{(5,20)}$

ALT serum concentrations were measured at the Fondazione IRCCS Clinical laboratory, "Alessandro Manzoni” Hospital Lecco, and Royal Free Hospital according to the IFCC standardized method performed at $37^{\circ} \mathrm{C}$ with the addition of pyrodoxalphosphate, ${ }^{(9,11)}$ on Roche Cobas c702 automatic analyzers using Roche reagents (Roche Diagnostics, Monza, Italy). The study plan was approved by the review board of the Fondazione. Liver damage biomarkers evaluation was approved by the competent ethics committees, and subjects gave written informed consent to the procedures.

\section{STATISTICAL ANALYSIS}

The choice of defining an updated ALT-URLIFCC by the 95 th percentile in healthy individuals without risk factors for liver disease was chosen based on $^{(21)}$ (1) nonnormal, highly right-skewed distribution of ALT; (2) lack of association of low ALT levels with disease; (3) high prevalence of the most common liver disease (NAFLD) in unselected healthy individuals; (4) most frequent use of ALT-URL-IFCC to screen for NAFLD; and (5) ample validation of this approach. ${ }^{(5-8)}$

Serum ALT levels were fitted with univariate and multivariate general linear models. Models were adjusted for confounding factors, as specified. Not normally distributed variables, including ALT levels, were log-transformed before entering the models. Receiver operating characteristic curves, in which the sensitivity was plotted against the false-positive rate (1-specificity) were generated to evaluate new ALT thresholds.

Statistical analyses were carried out using the JMP professional 15.0 (SAS Institute, Cary, NC) and $\mathrm{R}$ statistical analysis software version 4.0.2 (http:// www.R-project.org/). $P$ values $<0.05$ were considered statistically significant.

\section{Results}

\section{Ca' GRANDA-2018 COHORT}

The clinical features of the Ca' Granda-2018 cohort stratified by sex are presented in Table 1 . Participants were predominantly young adults; two-thirds of them were males and regular blood donors. Most had normal glucose, lipid and ferritin levels, and a minority were obese or had impaired fasting glucose. Arterial hypertension was more common, especially in males, with almost one-third being affected by this condition. A small fraction of participants, who were primarily included among those presenting at first donation, were reactive for $\mathrm{HBsAg}$ or anti-HCV antibodies by screening assays, but infection was not necessarily confirmed by supplemental serology and nucleic acid testing. The sex-specific distribution of ALT levels in the whole Ca' Granda-2018 cohort is presented in Table 2, upper panel, and in Supporting Fig. S3A.

\section{INDEPENDENT PREDICTORS OF ALT LEVELS IN HEALTHY INDIVIDUALS}

The independent predictors of ALT levels in the entire Ca' Granda-2018 cohort and in the subjects stratified by sex are given in Table 3 . As expected, the 
TABLE 2. DISTRIBUTION OF ALT SERUM ACTIVITY

(U/L) STRATIFIED BY SEX INTHE WHOLE CA'

GRANDA-2018 COHORT AND IN INDIVIDUALS

WITHOUT RISK FACTORS FOR LIVER DISEASE

ALT Percentile

\begin{tabular}{lcc}
\hline Whole Cohort & Males $(n=13,741)$ & Females $(n=7,555)$ \\
\hline 5 & 13 & 10 \\
25 & 18 & 14 \\
50 & 23 & 17 \\
75 & 30 & 21 \\
95 & 48 & 33
\end{tabular}

Without Risk Factors

for Liver Disease $\quad$ Males $(n=4,855) \quad$ Females $(n=4,330)$

\begin{tabular}{lll}
\hline 5 & 13 & 10 \\
25 & 17 & 13 \\
50 & 21 & 16 \\
75 & 27 & 20 \\
95 (URL) & 42 & 30
\end{tabular}

Note: The distribution of ALT percentiles was obtained in participants stratified by sex.

main predictor of ALT levels was male sex. Higher ALT values were very robustly associated with adiposity, as estimated by the BMI, but also with total cholesterol and ferritin levels. In the overall cohort and in males, there was also an independent association with glucose, triglycerides, and the presence of arterial hypertension. Younger age was also associated with ALT.

In the overall cohort and in females, there was a link between regular physical activity and a statistically significant, but quantitatively limited, increase in ALT. In the overall cohort and in males there was a protective association between a history of recreational cannabis use (tolerated in terms of suitability to donation when occurred more than 1 month apart from the presentation) and ALT. There was no significant association between regular intake of alcoholic beverages or positive viral markers (in individuals presenting for the first evaluation for blood donation) and ALT.

\section{DEFINITION OF ALT-URL-IFCC}

In the $\mathrm{Ca}$ ' Granda-2018 cohort, 4,855 males (35.3\%) and 4,340 females (57.4\%) had no risk factors for liver disease (Table 1, middle panel). The distribution of ALT levels in these individuals without
TABLE 3. INDEPENDENT DETERMINANTS OF SERUM ALT LEVELS (LOG U/L) IN THE WHOLE CA' GRANDA-2018 COHORT OF 21,296 HEALTHY INDIVIDUALS, AND IN MALES/FEMALES SEPARATELY

\begin{tabular}{|c|c|c|c|}
\hline & Estimate & SEM & $P$ Value \\
\hline \multicolumn{4}{|l|}{ Overall Cohort, $n=21,296$} \\
\hline Sex, F & -0.103 & 0.003 & $6 * 10^{-238}$ \\
\hline Age, years & -0.001 & 0.002 & $2 * 10^{-10}$ \\
\hline $\mathrm{BMI}, \mathrm{kg} / \mathrm{m}^{2}$ & +0.022 & 0.001 & $2 * 10^{-159}$ \\
\hline Glucose, $10 \mathrm{mg} / \mathrm{dL}$ & +0.010 & 0.006 & 0.003 \\
\hline Triglycerides, 10 mg/dL & +0.004 & 0.003 & $5^{*} 10^{-16}$ \\
\hline Total cholesterol, 10mg/dL & +0.010 & 0.001 & $2 * 10^{-46}$ \\
\hline Ferritin, log ng/mL & +0.083 & 0.003 & $2 * 10^{-117}$ \\
\hline Arterial hypertension, yes & +0.012 & 0.003 & 0.0002 \\
\hline Physical activity, moderate/severe & +0.007 & 0.002 & 0.013 \\
\hline HBsAg or anti-HCV Ab, positive & +0.004 & 0.008 & 0.59 \\
\hline Recreational drug use, yes & -0.011 & 0.004 & 0.019 \\
\hline Regular alcohol drinking, yes & -0.006 & 0.004 & 0.16 \\
\hline \multicolumn{4}{|l|}{ Males, $n=13,741$} \\
\hline & Estimate & SEM & $P$ Value \\
\hline Age, years & -0.004 & 0.000 & $4 * 10^{-34}$ \\
\hline $\mathrm{BMI}, \mathrm{kg} / \mathrm{m}^{2}$ & +0.029 & 0.001 & $1 * 10^{-153}$ \\
\hline Glucose, mg/dL & +0.001 & 0.000 & 0.0001 \\
\hline Triglycerides, mg/dL & +0.0005 & 0.0004 & $1 * 10^{-16}$ \\
\hline Total cholesterol, mg/dL & +0.001 & 0.000 & $3^{*} 10^{-46}$ \\
\hline Ferritin, log ng/mL & +0.078 & 0.004 & $3 * 10^{-70}$ \\
\hline Arterial hypertension, yes & +0.012 & 0.003 & 0.001 \\
\hline Physical activity, moderate/severe & +0.001 & 0.003 & 0.79 \\
\hline HBsAg or anti-HCV Ab, positive & +0.018 & 0.011 & 0.13 \\
\hline Recreational drug use, yes & -0.015 & 0.005 & 0.005 \\
\hline Regular alcohol drinking, yes & +0.004 & 0.005 & 0.50 \\
\hline \multicolumn{4}{|l|}{ Females, $n=7,555$} \\
\hline & Estimate & SEM & $P$ Value \\
\hline Age, years & -0.001 & 0.003 & $3^{*} 10^{-7}$ \\
\hline $\mathrm{BMI}, \mathrm{kg} / \mathrm{m}^{2}$ & +0.014 & 0.001 & $2 * 10^{-28}$ \\
\hline Glucose, $10 \mathrm{mg} / \mathrm{dL}$ & +0.000 & 0.000 & 0.65 \\
\hline Triglycerides, 10 mg/dL & +0.000 & 0.000 & 0.97 \\
\hline Total cholesterol, 10 mg/dL & +0.010 & 0.002 & $3^{*} 10^{-7}$ \\
\hline Ferritin, log ng/ml & +0.070 & 0.006 & $2 * 10^{-29}$ \\
\hline Arterial hypertension, yes & +0.010 & 0.006 & 0.097 \\
\hline Physical activity, moderate/severe & +0.018 & 0.005 & $9 * 10^{-5}$ \\
\hline HBsAg or anti-HCV Ab, positive & +0.004 & 0.008 & 0.75 \\
\hline Recreational drug use, yes & -0.006 & 0.008 & 0.48 \\
\hline Regular alcohol drinking, yes & -0.009 & 0.005 & 0.098 \\
\hline
\end{tabular}

Note: $P$ values were determined at multivariate generalized linear models, adjusted for the confounders specified in the table.

risk factors stratified by sex is presented in Table 2 (lower panel) and Supporting Fig. S3B. The updated ALT-URL-IFCC (95th percentile) were identified 
at $42 \mathrm{U} / \mathrm{L}$ (95\% confidence interval $[\mathrm{CI}]=40-43)$ in males, and $30 \mathrm{U} / \mathrm{L}(95 \% \mathrm{CI}=29-32)$ in females. Although a relation between age and ALT levels was found, the extent of the variation in terms of $\mathrm{U} / \mathrm{L}$ per year was limited (Table 3), and the introduction of age-specific cutoffs was not justified. Sensitivity analyses are presented in the Supporting Results and Supporting Table S3.

ALT predictors in participants without risk factors for liver disease were similar to those observed in the overall cohort and are reported in the Supporting Results and Supporting Tables S4 and S5.

A detailed characterization of participants with persistently increased ALT levels, and of the underlying causes of liver disease, is reported in the Supporting Results and in Supporting Table S6.

\section{IMPACT OF UPDATED ALT-URL- IFCC ON THE PREDICTION OF FATTY LIVER DISEASE}

The clinical features of the Liver-Bible-2020 cohort are presented in Table 1, lower panel. Steatosis was detected in 510 (68.6\%), and significant fibrosis in 16 (2.2\%). The diagnostic accuracy of the new ALT-URLIFCC versus old ALT-URL-IFCC for discriminating liver damage is presented in Table 4. Although the previously adopted ALT-URL-IFCC was not helpful in the detection of both steatosis or fibrosis, the new ALT-URL-IFCC was associated with both steatosis $(P=0.001)$ and with significant fibrosis $(P=0.021)$, conferring the possibility to detect significant fibrosis, despite with low accuracy (area under the receiver operating characteristic curve $[$ AUROC $]=0.61$ ). The advantage of the updated ALT-URL-IFCC was conferred by the higher sensitivity to detect liver damage.

In the Liver-Bible-2020 cohort, ALT levels were proportional to hepatic fact content, as estimated by CAP (estimate $=18.6 \pm 3.6, P=2^{*} 10^{-7}$ ), and to liver stiffness (estimate $=0.88 \pm 0.11, P=6^{*} 10^{-15}$ ). The best ALT cutoffs for steatosis detection were at $35 \mathrm{U} / \mathrm{L}$ in males (sensitivity $=0.36$, specificity $=0.80$ ) and $22 \mathrm{U} / \mathrm{L}$ in females (sensitivity $=0.59$, specificity $=0.69$ ). Significant fibrosis was not detected in females. The best cutoffs for fibrosis detection were at $27 \mathrm{U} / \mathrm{L}$ in males (sensitivity $=0.94$, specificity $=0.45$ ). However, 24.6\% of individuals in the $\mathrm{Ca}$ ' Granda-2018 cohort (31.4\% of those with and $15.8 \%$ of those without risk factors, $P<0.0001$ ) had ALT $>27 \mathrm{U} / \mathrm{L}$. 
In the Lecco cohort, which was not selected for dysmetabolism, the updated ALT-URL-IFCC predicted abnormal LSM (odds ratio $[\mathrm{OR}]=2.14,1.16$ $3.95 ; P=0.014$; sensitivity $=0.24$, specificity $=0.87$; positive likelihood ratio $(\mathrm{LR}+)=1.87$, negative likelihood ratio $(\mathrm{LR}-)=0.87)$, and significant liver fibrosis $(\mathrm{OR}=2.32,1.02-5.31 ; P=0.045$; sensitivity $=0.26$, specificity $=0.87 ; \mathrm{LR}+=1.98, \mathrm{LR}-=0.85)$, although with limited accuracy. The thresholds for individuals without dysmetabolism and risk factors for liver disease in the Lecco cohort were almost coincident to the updated ALT-URL-IFCC (i.e., 43 U/L [95\% CI 40-55] in males $[\mathrm{n}=176]$ and $32 \mathrm{U} / \mathrm{L}$ [95\% CI 30$35]$ in females $[\mathrm{n}=193]$.

The accuracy of the updated ALT-URL-IFCC for detecting liver disease in clinical cohorts was moderate to high (AUROC $=0.78-0.93$ ) and is reported in the Supporting Results and Supporting Tables S7 and S8.

\section{Discussion}

In this study, we updated the healthy ranges for ALT with the new standardized IFCC methodology and the changing epidemiology of liver disease. NAFLD is now the leading cause of liver disease, and it affects at least one-fourth of the subjects enrolled as healthy volunteers in clinical trials, which leads to some concern on the safety of participants and validity of study findings. ${ }^{(2)}$ There is currently low awareness of the differences between the methodologies available to measure ALT levels, and new uncertainties concerning the thresholds to be adopted in general practice to screen for liver disease. Although more accurate methods to discriminate chronic liver disease and fibrosis are available, ${ }^{(15,17)}$ ALT still represent the most used liver damage biomarker worldwide. ${ }^{(23)}$ Furthermore, noninvasive scores of fibrosis still rely on the evaluation of ALT levels, and by introducing a source of heterogeneity, the concomitant use of two different measurement methods and different definitions of the reference populations may negatively affect the accuracy of such approaches. ${ }^{(24)}$ The new IFCC methodology of ALT measurement was developed to improve the standardization among and within the different labs and clinical settings, but it is not yet widely adopted worldwide. Our main aim was to pragmatically set thresholds able to identify apparently healthy individuals at higher risk of liver disease, and in particular NAFLD, in the general population. We therefore repeated with the proposed analytic method and in the current epidemiological situation the same approach successfully used 20 years ago. ${ }^{(5)}$ We used the 95th percentile, as we were specifically interested in the upper bound of reference range, as a potential indicator of liver injury. ${ }^{(5)}$

Revised ALT-URL-IFCC were 42/30 in M/F, higher than the healthy limits determined by the same approach at the same center using an old, nonstandardized method (30/19), ${ }^{(5)}$ but lower than the currently accepted reference range for the IFCC standardized method (59/41). ${ }^{(11)}$ The main advantage of the proposed approach is that by the careful characterization of the risk factors in a large cohort, we were able to exclude from the reference population those at higher risk of liver disease and NAFLD, thereby providing "healthy limits" with improved ability to discriminate these conditions. The choice of the revised ALT-URL-IFCC was corroborated by the results of several sensitivity analyses. We also showed that the choice of the revised ALT-URL-IFCC was consistent with data derived from an independent cohort from the same geographical area.

The major ALT predictors in healthy individuals were male sex and increased adiposity. We also found a strong association with dyslipidemia, including high level of cholesterol and triglycerides, and ferritin levels, which reflect both insulin resistance and hepatic iron stores. ${ }^{(25-27)}$ Hypertension and high glucose levels were also independent predictors. Overall, these data suggest that metabolic risk factors influencing hepatic fat accumulation represent the major determinant of ALT levels in healthy individuals. In keeping with this notion, we recently showed that inherited genetic variants influencing hepatic fat accumulation are the main determinant of serum ALT levels in the general population. ${ }^{(28)}$ The determinants of ALT levels within the normal range, including recent physical activity and age, are discussed in detail in the Supporting Discussion. ${ }^{(5,8,29-33)}$

Due to the higher sensitivity compared with the thresholds previously proposed for the IFCC method, ${ }^{(11)}$ the revised ALT-URL-IFCC rendered it possible to discriminate the presence of NAFLD and of significant liver fibrosis in individuals with dysmetabolism. The ability to predict significant fibrosis was confirmed in an independent cohort of unselected blood donors. However, in keeping with previous literature data, ${ }^{(34-37)} 82 \%$ of individuals with steatosis and $54 \%$ of those with significant fibrosis had normal 
ALT levels, even when the definition was based on the updated thresholds, which are lower than those currently advised. In the setting of dysmetabolism, an even lower threshold at $27 \mathrm{U} / \mathrm{L}$ was more accurate to discriminate the presence of significant fibrosis. However, this lower threshold with higher sensitivity to detect subtle liver damage would not be appropriate for the overall population because of the very low specificity, ${ }^{(38)}$ as they would trigger an unacceptable amount of unnecessary follow-up tests and investigations.

This study has some limitations. These include the lack of direct assessment of the presence of fatty liver and fibrosis by FibroScan in the whole cohort. ${ }^{(17)}$ However, we could evaluate a comprehensive panel or risk factors and viral markers. Although the concomitant assessment in diagnostic algorithms of other noninvasive predictors may improve the rate of liver disease diagnosis and classification, ${ }^{(39)}$ the correct definition of the most appropriate ALT threshold to trigger further investigations remains of paramount importance. In addition, we could not compare ALT levels, determined by both the old and the new methodology, in the same samples. Furthermore, the updated ALT-URL-IFCC cannot be generalized to the developmental age group ${ }^{(40)}$ the elderly, populations of different ethnicity, or special populations.

In conclusion, we used a validated approach to update the healthy ranges of ALT determination by the new IFCC standardized methodology. In both sexes, the revised ALT-URL-IFCC (42/30 U/L in $\mathrm{M} / \mathrm{F}$ ) were about $30 \%$ lower than those calculated in initial studies, ${ }^{(11)}$ but higher than those recommended for the old, nonstandardized method. ${ }^{(5)}$ These revised ALT-URL-IFCC were able to identify individuals with liver injury related to dysmetabolism (e.g., NAFLD/MAFLD), which supports their use in general clinical practice. However, among patients with clinical NAFLD, ALT alone had limited accuracy to stratify the risk of progressive disease, ${ }^{(34-37)}$ and additional testing is required. ${ }^{(39,41)}$ Remarkably, the IFCC standardized methodology is not yet universally adopted, as the old, nonstandardized method is still in use in several laboratories. The existence of two methodologies producing substantially different ALT results is of concern, as it may lead to wrong diagnosis and clinical errors. While waiting for a harmonization of laboratory techniques, clinicians should be aware of which method has been used in each patient and apply the appropriate threshold.
Patient and Public Involvement: It was not appropriate or possible to involve patients or the public in the design, conduct, reporting, or dissemination of plans of our research. However, liver disease screening in healthy blood donors was disseminated and conducted at the Fondazione IRCCS $\mathrm{Ca}^{\prime}$ Granda Ospedale Maggiore Policlinico Blood Bank in concert with the blood donors' association ("Fondazione Amici del Policlinico").

Data Availability Statement: The data that support the findings of this study are available from D.P. and L.V., upon reasonable request.

\section{REFERENCES}

1) Younossi $Z$, Henry L. Contribution of alcoholic and nonalcoholic fatty liver disease to the burden of liver-related morbidity and mortality. Gastroenterology 2016;150:1778-1785.

2) Younossi ZM, Koenig AB, Abdelatif D, Fazel Y, Henry L, Wymer M. Global epidemiology of nonalcoholic fatty liver diseasemeta-analytic assessment of prevalence, incidence, and outcomes. Hepatology 2016;64:73-84.

3) Goldberg D, Ditah IC, Saeian K, Lalehzari M, Aronsohn A, Gorospe EC, et al. Changes in the prevalence of hepatitis $\mathrm{C}$ virus infection, nonalcoholic steatohepatitis, and alcoholic liver disease among patients with cirrhosis or liver failure on the waitlist for liver transplantation. Gastroenterology 2017;152:1090-1099.e1091.

4) Kim WR, Flamm SL, Di Bisceglie AM, Bodenheimer HC; Public Policy Committee of the American Association for the Study of Liver Disease. Serum activity of alanine aminotransferase (ALT) as an indicator of health and disease. Hepatology 2008;47:1363-1370.

5) Prati D, Taioli E, Zanella A, Torre ED, Butelli S, Del Vecchio E, et al. Updated definitions of healthy ranges for serum alanine aminotransferase levels. Ann Intern Med 2002;137:1-10.

6) Kim KN, Joo J, Sung HK, Kim CH, Kim H, Kwon YJ. Associations of serum liver enzyme levels and their changes over time with all-cause and cause-specific mortality in the general population: a large-scale national health screening cohort study. BMJ Open 2019;9:e026965.

7) Park JH, Choi J, Jun DW, Han SW, Yeo YH, Nguyen MH. Low alanine aminotransferase cut-off for predicting liver outcomes; a nationwide population-based longitudinal cohort study. J Clin Med 2019;8:1445.

8) Lee JK, Shim JH, Lee HC, Lee SH, Kim KM, Lim Y-S, et al. Estimation of the healthy upper limits for serum alanine aminotransferase in Asian populations with normal liver histology. Hepatology 2010;51:1577-1583.

9) Schumann G, Bonora R, Ceriotti F, Ferrero CA, Franck PFH, Javier CF, et al. IFCC primary reference procedures for the measurement of catalytic activity concentrations of enzymes at 37 degrees C. International Federation of Clinical Chemistry and Laboratory Medicine. Part 4. Reference procedure for the measurement of catalytic concentration of alanine aminotransferase. Clin Chem Lab Med 2002;40:718-724.

10) Infusino I, Schumann G, Ceriotti F, Panteghini M. Standardization in clinical enzymology: a challenge for the theory of metrological traceability. Clin Chem Lab Med 2010;48:301-307.

11) Ceriotti F, Henny J, Queralto J, Ziyu S, Özarda Y, Chen B, et al. Common reference intervals for aspartate aminotransferase (AST), alanine aminotransferase (ALT) and gamma-glutamyl transferase (GGT) in serum: results from an IFCC multicenter study. Clin Chem Lab Med 2010;48:1593-1601. 
12) Estes C, Anstee QM, Arias-Loste MT, Bantel H, Bellentani S, Caballeria J, et al. Modeling NAFLD disease burden in China, France, Germany, Italy, Japan, Spain, United Kingdom, and United States for the period 2016-2030. J Hepatol 2018;69:896-904.

13) Singh S, Allen AM, Wang Z, Prokop LJ, Murad MH, Loomba R. Fibrosis progression in nonalcoholic fatty liver vs nonalcoholic steatohepatitis: a systematic review and meta-analysis of pairedbiopsy studies. Clin Gastroenterol Hepatol 2015;13:643-654. e641-649; quiz e639-e640.

14) Pelusi S, Cespiati A, Rametta R, Pennisi G, Mannisto V, Rosso $\mathrm{C}$, et al. Prevalence and risk factors of significant fibrosis in patients with nonalcoholic fatty liver without steatohepatitis. Clin Gastroenterol Hepatol 2019;17:2310-2319.e2316.

15) European Association for the Study of the Liver. European Association for the Study of Diabetes, European Association for the Study of Obesity. EASL-EASD-EASO Clinical Practice Guidelines for the management of non-alcoholic fatty liver disease. J Hepatol 2016;64:1388-1402.

16) Karlas T, Petroff D, Sasso M, Fan J-G, Mi Y-Q, de Lédinghen $\mathrm{V}$, et al. Individual patient data meta-analysis of controlled attenuation parameter (CAP) technology for assessing steatosis. J Hepatol 2017;66:1022-1030.

17) Maida M, Macaluso FS, Salomone F, Petta S. Non-invasive assessment of liver injury in non-alcoholic fatty liver disease: a review of literature. Curr Mol Med 2016;16:721-737.

18) Bazerbachi F, Haffar S, Wang Z, Cabezas J, Arias-Loste MT, Crespo J, et al. Range of normal liver stiffness and factors associated with increased stiffness measurements in apparently healthy individuals. Clin Gastroenterol Hepatol 2019;17:54-64.e51.

19) Colli A, Fraquelli M, Prati D, Riva A, Berzuini A, Conte D, et al. Deciding on interferon-free treatment for chronic hepatitis $C$ : updating liver stiffness cut-off values to maximize benefit. PLoS One 2016;11:e0164452.

20) Kleiner DE, Brunt EM, Van Natta M, Behling C, Contos MJ, Cummings OW, et al. Design and validation of a histological scoring system for nonalcoholic fatty liver disease. Hepatology 2005;41:1313-1321.

21) Whyte MB, Kelly $P$. The normal range: it is not normal and it is not a range. Postgrad Med J 2018;94:613-616.

22) Takyar V, Nath A, Beri A, Gharib AM, Rotman Y. How healthy are the "Healthy volunteers"? Penetrance of NAFLD in the biomedical research volunteer pool. Hepatology 2017;66:825-833.

23) Pacifico L, Ferraro F, Bonci E, Anania C, Romaggioli S, Chiesa C. Upper limit of normal for alanine aminotransferase: quo vadis? Clin Chim Acta 2013;422:29-39.

24) Hagstrom $H$, Talback $M$, Andreasson A, Walldius G, Hammar N. Repeated FIB-4 measurements can help identify individuals at risk of severe liver disease. J Hepatol 2020;73:1023-1029.

25) Dongiovanni P, Fracanzani AL, Fargion S, Valenti L. Iron in fatty liver and in the metabolic syndrome: a promising therapeutic target. J Hepatol 2011;55:920-932.

26) Valenti L, Dongiovanni P, Fargion S. Diagnostic and therapeutic implications of the association between ferritin level and severity of nonalcoholic fatty liver disease. World J Gastroenterol 2012;18:3782-3786.

27) Ryan JD, Armitage AE, Cobbold JF, Banerjee R, Borsani O, Dongiovanni $\mathrm{P}$, et al. Hepatic iron is the major determinant of serum ferritin in NAFLD patients. Liver Int 2018;38:164-173.

28) Jamialahmadi O, Mancina RM, Ciociola E, Tavaglione F, Luukkonen PK, Baselli G, et al. Exome-wide association study on alanine aminotransferase identifies sequence variants in the GPAM and APOE associated with fatty liver disease. Gastroenterology 2021;160:1634-1646.e7.

29) Lazo M, Selvin E, Clark JM. Brief communication: clinical implications of short-term variability in liver function test results. Ann Intern Med 2008;148:348-352.

30) Kariv R, Leshno M, Beth-Or A, Strul H, Blendis L, Kokia E, et al. Re-evaluation of serum alanine aminotransferase upper normal limit and its modulating factors in a large-scale population study. Liver Int 2006;26:445-450.

31) Völzke H, Alte D, Ittermann T, Schmidt CO, Rettig R, Mayerle $\mathrm{J}$, et al. Subjects with sonographical hepatic steatosis should be excluded from studies to establish upper reference levels of serum transaminases. Liver Int 2011;31:985-993.

32) Elinav E, Ben-Dov IZ, Ackerman E, Kiderman A, Glikberg F, Shapira Y, et al. Correlation between serum alanine aminotransferase activity and age: an inverted U curve pattern. Am J Gastroenterol 2005;100:2201-2204.

33) Turola E, Petta S, Vanni E, Milosa F, Valenti L, Critelli R, et al. Ovarian senescence increases liver fibrosis in humans and zebrafish with steatosis. Dis Model Mech 2015;8:1037-1046.

34) Fracanzani AL, Valenti L, Bugianesi E, Andreoletti M, Colli A, Vanni E, et al. Risk of severe liver disease in nonalcoholic fatty liver disease with normal aminotransferase levels: a role for insulin resistance and diabetes. Hepatology 2008;48:792-798.

35) Mofrad P, Contos MJ, Haque M, Sargeant C, Fisher RA, Luketic VA, et al. Clinical and histologic spectrum of nonalcoholic fatty liver disease associated with normal ALT values. Hepatology 2003;37:1286-1292.

36) Maximos M, Bril F, Portillo Sanchez P, Lomonaco R, Orsak B, Biernacki $\mathrm{D}$, et al. The role of liver fat and insulin resistance as determinants of plasma aminotransferase elevation in nonalcoholic fatty liver disease. Hepatology 2015;61:153-160.

37) Browning JD, Szczepaniak LS, Dobbins R, Nuremberg P, Horton JD, Cohen JC, et al. Prevalence of hepatic steatosis in an urban population in the United States: impact of ethnicity. Hepatology 2004;40:1387-1395.

38) Ruhl CE, Everhart JE. Upper limits of normal for alanine aminotransferase activity in the United States population. Hepatology 2012;55:447-454.

39) Dillon JF, Miller MH, Robinson EM, Hapca A, Rezaeihemami $\mathrm{M}$, Weatherburn C, et al. Intelligent liver function testing (iLFT): a trial of automated diagnosis and staging of liver disease in primary care. J Hepatol 2019;71:699-706.

40) Kang Y, Park S, Kim S, Koh H. Normal serum alanine aminotransferase and non-alcoholic fatty liver disease among Korean adolescents: a cross-sectional study using data from KNHANES 2010-2015. BMC Pediatr 2018;18:215.

41) Newsome PN, Cramb R, Davison SM, Dillon JF, Foulerton M, Godfrey EM, et al. Guidelines on the management of abnormal liver blood tests. Gut 2018;67:6-19.

Author names in bold designate shared co-first authorship.

\section{Supporting Information}

Additional Supporting Information may be found at onlinelibrary.wiley.com/doi/10.1002/hep4.1794/suppinfo. 